\title{
Meshless Parameterization for Dimensional Reduction Integrated in 3D Voxel Reconstruction Using a Single PC
}

\author{
Yunli Lee, Dongwuk Kyoung, and Keechul Jung* \\ School of Media, College of Information Technology, Soongsil University, \\ Seoul, South Korea \\ \{yunli, kiki227, kcjung\}@ssu.ac.kr \\ http://hci.ssu.ac.kr
}

\begin{abstract}
Shape-From-Silhouettes (SFS) is one of the most popular ideas for reconstructing the $3 \mathrm{D}$ voxel of an object from silhouettes images. This paper presents a method based SFS and pre-computing methods for 3D voxel reconstruction using a single PC. This method is reduced the memory usage. Towards this approach, a meshless parameterization for dimensional reduction is integrated in the process to obtain object representation in 2D form. Since the meshless parameterization requires the solution of large linear system on a whole 3D voxel, by taking the advantages of $3 \mathrm{D}$ voxel reconstruction process, the meshless parameterization is computed locally. The proposed method is able to implement an optimize system and utilize in various applications.
\end{abstract}

Keywords: Meshless parameterization, dimensional reduction, 3D voxel reconstruction, shape from silhouettes, single PC.

\section{Introduction}

$3 \mathrm{D}$ voxel reconstruction using multiple-views of silhouette images is an active research topic in 3D video, 3D animation, 3D gesture recognition and etc. The 3D voxel reconstruction is referred to the problem of extracting the parameters of an object from a set of sequence images. Visual Hull (VH) construction and ShapeFrom-Silhouettes (SFS) approximates the shape using silhouettes images have been proposed in last two decades [1,2]. SFS is one of most popular approach for shape estimation since it has many advantages for estimating 3D shape. For better shape estimation, multiple cameras are used to increase the number of silhouette images.

The 3D voxel reconstruction involves many operations therefore PC clusters are used in order to perform a fast processing speed. However, the PC clusters need high cost, high speed network and synchronizing multiple-view images. This motivates us proposed a pre-computing method using look-up table for 3D voxel reconstruction in a single PC. The proposed system consists of one PC, four web cameras and a graphic card. This method optimizes the performance and speed for $3 \mathrm{D}$ voxel reconstruction using a single PC.

\footnotetext{
${ }^{*}$ Corresponding author.
} 
In addition, the interest of dimensional reduction of 3D voxel has grown rapidly. The 3D voxel is rich of information that supports the various kinds of applications. However, this information causes high computational time for real time performance. Dimensional reduction becomes very important to reduce the curse of dimensionality. There are various approaches of dimension reduction such as Principal Component Analysis (PCA), Linear Discriminant Analysis (LDA), Multi-Dimensional Scaling (MDS) and etc $[3,10,11]$. These techniques are popular for pattern recognition areas. Yet, we chose Meshless Parameterization, as a technique for dimensional reduction of the reconstructed 3D voxel. The meshless parameterization is well known for parameterizing and triangulating a single patch for unorganized point sets [4-8].

The basic idea of meshless parameterization is to map the points into some convex parameter domain in the plane. This method is mapping independently in any given topological structure. However, this method requires a solution of a large linear system and the complexity of linear system is increased rapidly when the number of $3 \mathrm{D}$ voxel increased. Therefore, in this paper, we are adopted the advantages of the 3D voxel reconstruction, the meshless parameterization is computed locally within the process of generating the 3D voxel. This approach is able to overcome the linear system complexity. On top of this, the operations for number of neighborhoods and weight computation are performed faster.

An overview of our proposed system is illustrated in Fig. 1. In this paper, the 3D voxel is reconstructed using SFS method. The silhouettes images are extracted from four web cameras using a single PC. In the process of generating 3D voxel, meshless parameterization is computed for every three base plane slices. At the end of process, the system results a $3 \mathrm{D}$ voxel object and a $2 \mathrm{D}$ pixel object representation.

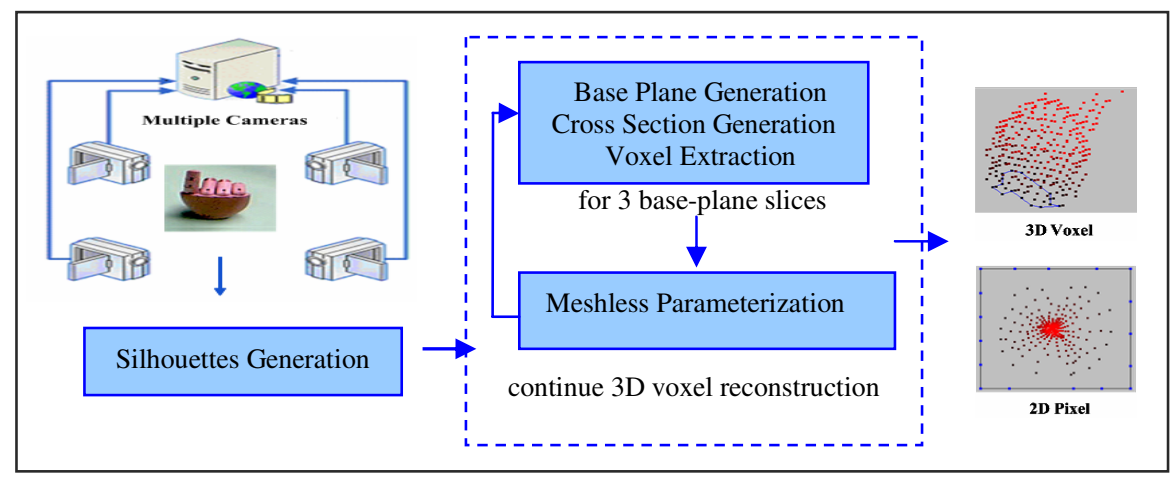

Fig. 1. The overview of proposed system

Shape-From-Silhouettes method for 3D voxel reconstruction using pre-computing approach is described in section 2. Section 3 explains the basic idea of meshless parameterization. The meshless parameterization towards the $3 \mathrm{D}$ voxel reconstruction in order to solve the large linear system is presented in section 4. The experimental results of the proposed system are elaborated in section 5. Conclusions and proposed future work are presented in section 6. 


\section{3D Voxel Reconstruction}

Matsuyama et al. [1] presented a real-time dynamic 3D object shape reconstruction method using sequential processes: silhouettes image generation (SIG), base-plane projection (BPP), parallel-plane projection (PPP) and intersection (INT). They are introduced a forward computation of all silhouettes pixels for $3 \mathrm{D}$ voxel generation using pc clusters approach. However, this process requires much computational time. We adopted the method of Shape-From-Silhouettes (SFS) to reconstruct the 3D object. However, we propose a fast and efficient method which runs on a PC, for 3D voxel reconstruction using pre-computing of 2D silhouettes image for one slice of plane and stored into a look-up table. Section 2.1 describes the overview of general method of SFS for 3D voxel reconstruction and section 2.2 explains our proposed approach of pre-computing.

\subsection{Basic Method of Shape-From-Silhouettes}

Shape-From-Silhouettes is implemented in proposed system. In this paper, the 3D object is reconstructed by volume intersection. To realize efficient volume intersection, the plane-based volume intersection method is introduced. The 3D voxel space is partitioned into a group of parallel planes and the cross section of the 3D object volume on each plane is reconstructed. Then, we devised the plane-to-plane perspective projection algorithm to realize efficient plane-to-plane projection computation. PC cluster is introduced to realize real-time processing. Fig. 2 shows a forward computation of all silhouettes pixels for 3D voxel generation on PC cluster.

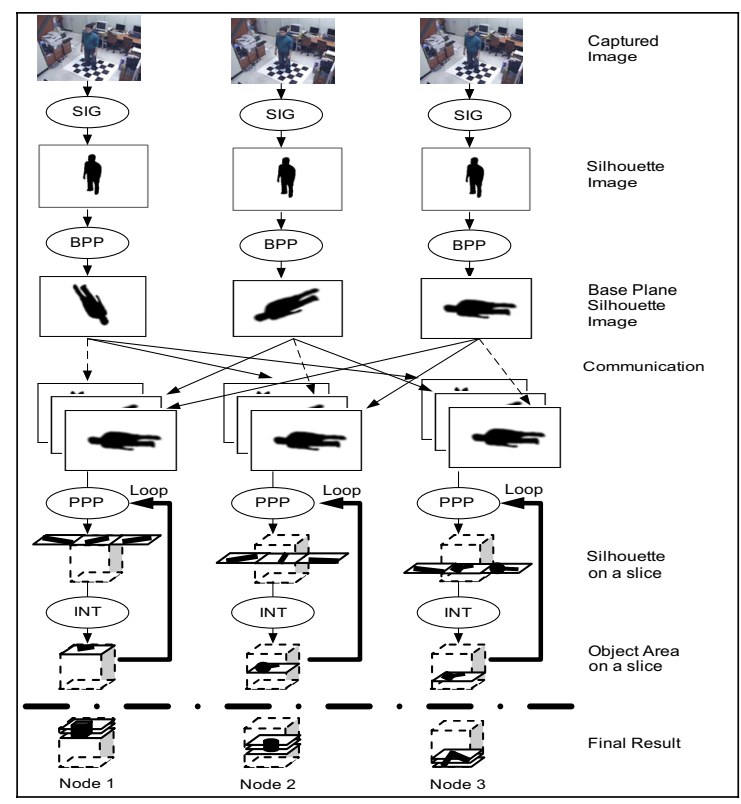

Fig. 2. 3D voxel reconstruction based on silhouettes pixel using PC clusters 


\subsection{Pre-computing Method}

The 3D voxel reconstruction consists of four steps: silhouettes generation, plane generation, cross section, and voxel extraction. It is represented in volumetric form. The silhouettes are generated using background subtraction and shadow detection from the color difference [2]. The proposed approach only relies on volumetric voxel data of the object in order to infer shape information. The threshold value of RGB color is derived to subtract the background and foreground images. The silhouette generation consists of two methods: first, compute the distance in order to separate a silhouette pixel and background pixel, and second, compute the difference to separate the shadow and non-shadow.

We present a fast and efficient method of 3D voxel reconstruction in order to reduce the memory storage and computational times. This approach used a computed plane data (pre-computing) and post-computing method. Fig. 3 shows the proposed method of pre-computing in 3D voxel reconstruction.

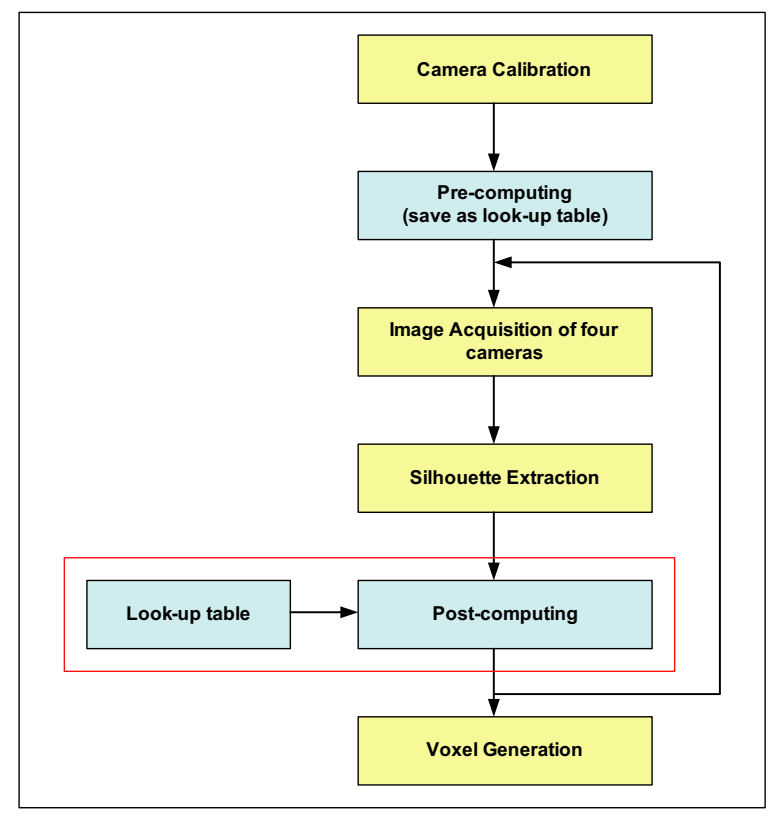

Fig. 3. Work flow of 3D voxel reconstruction using pre-computing approach

The projection of each voxel in the $3 \mathrm{D}$ volume is constrained on a $2 \mathrm{D}$ plane. The plane is $\mathrm{x}-\mathrm{y}$ axis and $\mathrm{z}$-axis is equal to zero. Then, the $2 \mathrm{D}$ silhouette points of this plane are pre-computed and saved in look-up table. The voxel reconstruction is fast and efficient with the assist of look-up table and post-computing process. In the proposed system, the relationship between a 3D point $(X, Y, Z)$ and its image projection of $2 \mathrm{D}$ point $\left(x_{1} / x_{3}, x_{2} / x_{3}\right)$ is given by the equation (1). 


$$
\left[\begin{array}{l}
x_{1} \\
x_{2} \\
x_{3}
\end{array}\right]=H\left[\begin{array}{l}
X \\
Y \\
1
\end{array}\right], H_{z}=\left[\begin{array}{lll}
h_{11} & h_{12} & h_{13} \\
h_{21} & h_{22} & h_{23} \\
h_{31} & h_{32} & h_{33}
\end{array}\right]=A\left[r_{1} r_{2}\left(r_{3} z+t\right)\right]
$$

As for $x_{1}, x_{2}$ and $x_{3}$ computation, the equation can be found in Table 1 . We can represent the general perspective projection from $3 \mathrm{D}$ voxel point $(X, Y, Z)$ to $2 \mathrm{D}$ point $\left(x_{1} / x_{3}, x_{2} / x_{3}\right)$. This process requires 22 multiplications, 14 additions and 2 divisions for each voxel point. However, for the equation (2) and (3), if the z-axis is zero for each voxel data, the parameter $A_{1}$, and $A_{2}$ are pre-computed and stored in look-up table. Therefore, this process reduces the computational times and only requires 5 multiplications, 5 additions and 2 divisions for each computation of all voxel data from plane 0 until plane $\mathrm{k}$. The data set for $\left(B_{1}, B_{2}, r_{31}, r_{32}, t_{3}, r_{33}\right)$ which derived from equation (2), (3) and (4) are also saved for each of web camera [12,13].

Table 1. List of equation for $x_{1}, x_{2}$ and $x_{3}$

\begin{tabular}{|c|c|}
\hline $\begin{aligned} x_{1}= & h_{11} \times X+h_{12} \times Y+h_{13} \\
& =\left(f_{x} \times r_{11}+c_{x} \times r_{31}\right) \times X+\left(f_{x} \times r_{12}+c_{x} \times r_{32}\right) \times Y \\
& +f_{x} \times\left(r_{13} \times Z+t_{1}\right)+c_{x} \times\left(r_{33} \times Z+t_{3}\right) \\
& =\left[\left(f_{x} \times r_{11}+c_{x} \times r_{31}\right) \times X+\left(f_{x} \times r_{12}+c_{x} \times r_{32}\right) \times Y\right. \\
& \left.+\left(f_{x} \times t_{1}+c_{x} \times t_{3}\right)\right]+\left(f_{x} \times r_{13}+c_{x} \times r_{33}\right) \times Z \\
& =A_{1}+B_{1} \times Z\end{aligned}$ & (2) \\
\hline $\begin{aligned} x_{2}= & h_{21} \times X+h_{22} \times Y+h_{23} \\
& =\left(f_{y} \times r_{21}+c_{y} \times r_{31}\right) \times X+\left(f_{y} \times r_{22}+c_{y} \times r_{32}\right) \times Y \\
& +f_{y} \times\left(r_{23} \times Z+t_{2}\right)+c_{y} \times\left(r_{33} \times Z+t_{3}\right) \\
& =\left[\left(f_{y} \times r_{21}+c_{y} \times r_{31}\right) \times X+\left(f_{y} \times r_{22}+c_{y} \times r_{32}\right) \times Y\right. \\
& \left.+\left(f_{y} \times t_{2}+c_{y} \times t_{3}\right)\right]+\left(f_{y} \times r_{23}+c_{y} \times r_{33}\right) \times Z \\
& =A_{2}+B_{2} \times Z\end{aligned}$ & (3) \\
\hline $\begin{aligned} x_{3} & =h_{31} \times X+h_{22} \times Y+h_{33} \\
& =r_{31} \times X+r_{32} \times Y+r_{33} \times Z+t_{3}\end{aligned}$ & (4) \\
\hline
\end{tabular}

\section{Meshless Parameterization}

The meshless parameterization method [4-8] is used to represent the $3 \mathrm{D}$ voxel data into 2D pixel representation which adopt good characteristics of convex combination such as fast computation and one-to-one mapping. We briefly describe the basic idea of meshless parameterization in the following sections. 


\subsection{Basic Idea}

Meshless parameterization is a 2D representation with some convex parameter where the one-to-one mappings of $3 \mathrm{D}$ voxel into $2 \mathrm{D}$ pixel without using mesh information [4-8]. The method is divided into two basic steps. First, map the boundary points $P_{B}$ into the boundary of domain $D$ plane. Then, the corresponding parameter points $U=$ $\left\{u_{n+1}, u_{n+2}, \ldots, u_{N}\right\}$ are laid around the domain $D$ counter-clockwise order. The chord length parameterization is used for the distribution of parameter points $U$. In this paper, we used the boundary-following algorithm to search and order the boundary points from the 3D voxel. The detail of the algorithm is described in section 3.2.

The second step, the interior points are mapped into the domain $D$ plane. However, before mapping, a neighborhood $p_{j}$ for each interior point in $P_{I}$ where the points are some sense closed by is chosen, and let $N_{\mathrm{i}}$ as a set of neighborhood points of $p_{i}$. In this case, a constant radius $r$ is chosen. The points that fall within the ball with radius $r$ are considered the neighborhood points of each interior point. Then, the reciprocal distance weights method is to compute the weight $\lambda_{i j}$ for each interior point $p i$. The parametric points for interior point's $u_{i}$ can be obtained by solving the linear system of $\mathrm{n}$ equations of the number of interior points, as shown in below equations (5) and (6).

$$
\begin{gathered}
u_{i}=\sum_{j \in N_{i}} \lambda_{i j} u_{j}, \quad i=1, \ldots, n . \\
u_{i}-\sum_{j \in N_{i} \cap P_{I}} \lambda_{i j} u_{j}=\sum_{j \in N_{i} \cap P_{B}} \lambda_{i j} u_{j}, i \in P_{I}
\end{gathered}
$$

From the equation, we rewrite the linear system in the form of $A u=b$, where $A$ is a matrix of weight $n \times n, u$ is parameter points and $b$ is the sum of neighbor points of $u$. The linear system can be written in the matrix form:

$$
\begin{gathered}
\text { where } A=\left[\begin{array}{cccc}
1 & -\lambda_{12} & \cdots & -\lambda_{1 n} \\
-\lambda_{21} & 1 & \cdots & -\lambda_{2 n} \\
\vdots & \vdots & \ddots & \vdots \\
-\lambda_{n 1} & -\lambda_{n 2} & \cdots & 1
\end{array}\right], \\
a_{i j}=\left\{\begin{array}{cc}
1 & , \quad j=i \\
-\lambda_{i j} & , \quad j \in N_{i} \\
0 & , \text { otherwise }
\end{array}, \text { and } u=\left[\begin{array}{c}
u_{1} \\
u_{2} \\
\vdots \\
u_{n}
\end{array}\right], b=\left[\begin{array}{c}
\sum_{j \in N_{1}} \lambda_{1 j} u_{j} \\
\sum_{j \in N_{2}} \lambda_{2 j} u_{j} \\
\vdots \\
\sum_{j \in N_{n}} \lambda_{n j} u_{j}
\end{array}\right] .\right.
\end{gathered}
$$

We use Gauss Elimination to compute the inverse of matrix A. Follows by solving the equation (7) and obtained the parametric value $U$ for all interior points, which are used to represent gesture data, and novel features are extracted for gesture recognition. 
Fig. 4 illustrated the result of 3D hand voxel data and the 2D representation of meshless parameterization. This meshless parameterization has two drawbacks: first, need to solve a large sparse linear system, and second, the neighborhood computation takes a linear search computation time. Both drawbacks have much computational times when the number of $3 \mathrm{D}$ voxel increases. This is not efficient for real-time application. In order to solve these problems, meshless parameterization is integrated in the 3D voxel reconstruction. The details approach of proposed method is presented in Section 4.

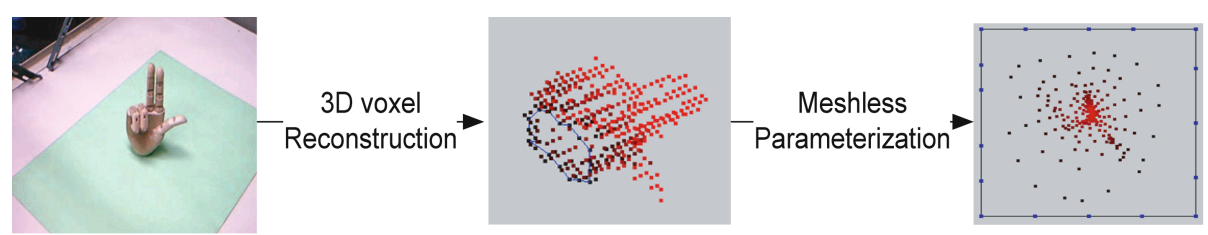

Fig. 4. Result of meshless parameterization for $3 \mathrm{D}$ hand voxel

\subsection{Neighborhood Points and Weight}

In this section, we describe a method to compute the neighborhood points and weight. The number of neighbor points are determined using a ball neighborhood with constant radius, r. The reciprocal distance weights method is to compute the weight, $\lambda_{i j}$ for each interior point $p i$. In this process, we reduced the computation time from all interior points into local interior points only. The equation (8) explains the method of choosing neighborhoods and weights. The choice of weights is positive $\lambda_{i j}$, for $j \in N_{i}$, such that $\sum_{j \in N_{i}} \lambda_{i j}=1$ where the parameter interior point, $u_{i}$ is some convex combination of its neighbor's $u_{j}$.

Let $N_{i}$ be the ball neighborhood

$N_{i}=\left\{j: 0<\left\|p_{j}-p_{i}\right\|<r\right\}$,

for some radius $r>0$

and let the $\lambda_{i j}$ be the reciprocal distance weights

$\lambda_{i j}=\frac{1}{\left\|p_{j}-p_{i}\right\|} / \sum_{k \in N_{i}} \frac{1}{\left\|p_{k}-p_{i}\right\|}$.

\subsection{Boundary-Following Algorithms}

The boundary-following algorithm is used for surface point's extraction and order boundary points in counterclockwise ordered. The 3D voxel data generation is extracted from the silhouette images and intersection points. The 3D voxel contains the whole volume of the 3D object information. The voxel data is extracted without proper ordered. In meshless parameterization, it only needs the $3 \mathrm{D}$ surface points instead of the whole volume. The boundary-following algorithm [9] is widely used on 
the boundary extraction on 2D image. Thanks to the base plane generation for 3D voxel which is the slice basis, it can be treated as a 2D domain image. The boundary of a connected component $\mathrm{S}$ is the set of pixels of $S$ that are adjacent to $\hat{S}$ is defined in 2D domain image. A simple local operation may be used to find pixels on the boundary. In this meshless parameterization, it wants to track pixels on the boundary in counterclockwise order. The details and simple boundary-following algorithm is given as follows:

1. Find the starting pixel $s \in S$ for the region using a systematic scan, say from left to right and from bottom to top of the image

2. Let the current pixel in boundary tracking be denoted by $c$. Set $c=s$ and let the 4-neighbor to the west of $s$ be $b \in \hat{S}$.

3. Let the eight 8-neighbors of $c$ starting with $b$ in counterclockwise order be $n_{1}, n$ $2, \ldots, n_{8}$. Find $n_{i}$, for the first $i$ that is in $S$.

4. Set $c=n_{i}$ and $b=n_{i}-1$.

5. Repeat steps 3 and 4 until $c=s$.

This process is very important to determine the boundary points. The first starting point of the boundary points is at the left most bottom position. The order of the boundary points sequence is counterclockwise ordered.

\section{Meshless Parameterization in 3D Voxel Reconstruction}

The objective of the proposed system is to represent the $3 \mathrm{D}$ voxel into $2 \mathrm{D}$ pixel representation that is useful for object recognition or related applications. In the same time, the integration of meshless parameterization in 3D voxel reconstruction reduced the computational complexity and memory storage. The overall proposed system of meshless parameterization for dimensional reduction integrated in pre-computing 3D voxel reconstruction using a single $\mathrm{PC}$ is described as following algorithm and Fig. 5:

1. Precompute the 2D silhouettes points correspond to $3 D$ voxel and store in look-up table

2. Synchronized the multiple images by taken simultaneously with multiple cameras

3. Extract the silhouette by background subtraction based on the RGB color threshold

4. Reconstruct the $3 D$ voxel data volume using post-computing based on look-up table

A. Every 3 slices compute neighbor points and weight for local meshless parameterization

5. Repeat step 4 until complete reconstruct $3 D$ voxel

The existing method of meshless parameterization requires the computation of the 2D parameterization to solve a large linear system. In order to solve the large sparse linear system, an iterative solver such as Gauss-Seidel or Krylov subspace methods [8] is used. Our proposed method can be overcome the large linear system using local $3 \mathrm{D}$ voxel for meshless parameterization process. This local approach of meshless parameterization is introduced during the process of reconstructing the $3 \mathrm{D}$ voxels.

The 3D reconstruction method is based on shape from silhouettes and generates the $3 \mathrm{D}$ voxel plane per plane. Original method of meshless parameterization of 3D voxels into $2 \mathrm{D}$ representation is gathered all the $3 \mathrm{D}$ voxels points and split them into 2 


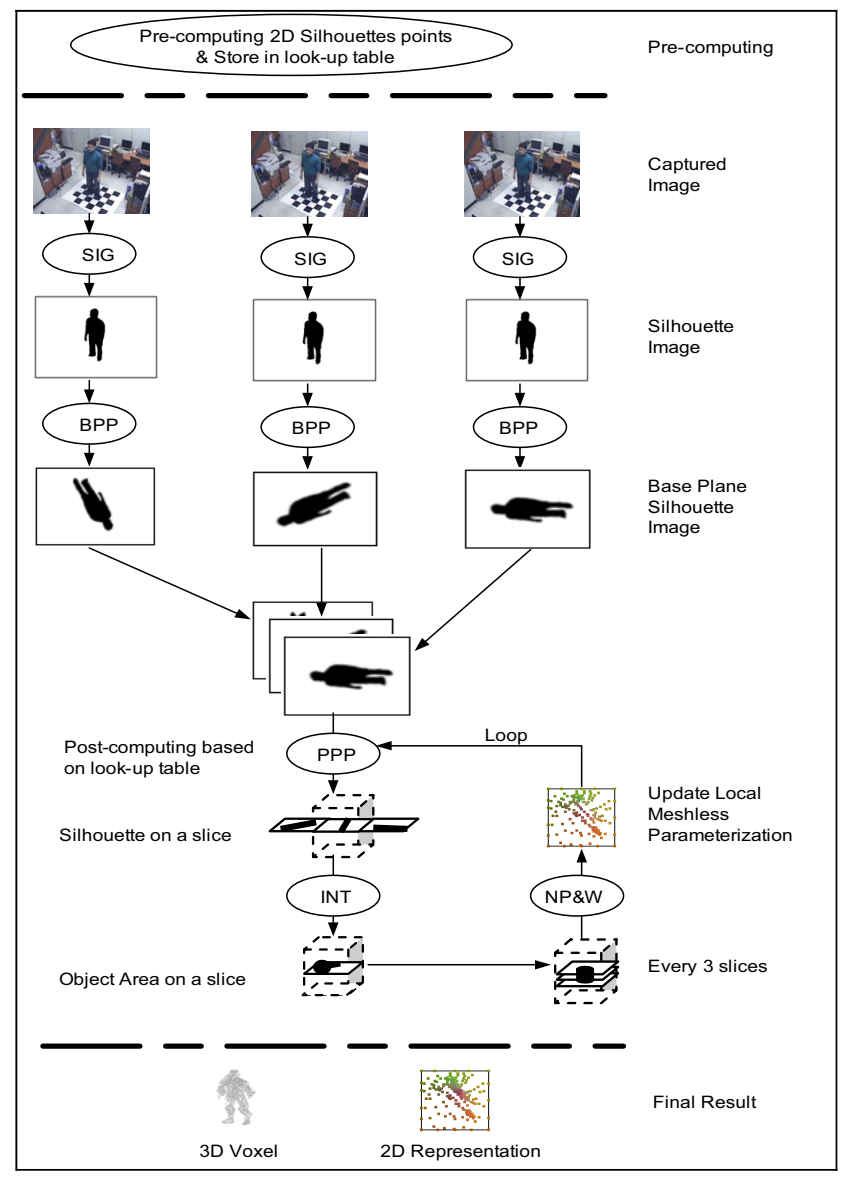

Fig. 5. The overall process of proposed system

disjoint sets. This process will generate a large sparse linear system during 2D parameterization computation. In addition, the process time of neighborhood computation increases rapidly according to number of voxel points. Therefore, we are introduced meshless parameterization integrated in the process of reconstructing the $3 \mathrm{D}$ object.

Fig. 5 shows the process of 3D reconstruction, the voxels are generated plane by plane. The first plane generation is equivalent to the boundary points one of two disjoints subsets of points for meshless parameterization. Therefore the boundary points are discriminated easily from the interior points. Only in the first plane, the boundary points are ordered in counter-clockwise order using boundary following algorithm. The process is carried out for the next plane of $3 \mathrm{D}$ voxels and this voxels are stored as interior points. The meshless parameterization using local 3D voxel is referred as partial simultaneously compute the 2D parameterization whenever there are three consequent of planes that generated in $3 \mathrm{D}$ reconstruction from bottom to upper plane. This process of meshless parameterization is illustrated as following pseudocode: 
1. Assume the first plane generation of $3 D$ voxels data as boundary points

2. Ordered the boundary points using boundary-following algorithm

3. Map the boundary points into $2 D$ domain using chord-length parameter

4. Obtained the two consequence planes of $3 D$ voxels data as interior points

5. Compute the neighbor points and weights

6. Solve the linear system to compute and update the interior points parameter

7. Treat the $2 D$ parameter points of first plane from interior points as map boundary points in 2D domain

8. Obtained the following plane and remain the last plane of $3 D$ voxel as interior points

9. Repeat the step 5 to 9 until last plane generation

\section{Experimental Results}

In this paper, we proposed two methods and integrated into a system. The first method is the pre-computing for $3 \mathrm{D}$ voxel reconstruction, and the second method is meshless parameterization for dimension reduction. Four cameras are placed towards a defined workspace to capture the object. The system is processed with a personal computer (Intel® Xeon ${ }^{\mathrm{TM}}$ CPU 3.20GHz, 2.56GB RAM, Windows XP OS).

We have tested on overall performance for general method, look-up table and our proposed method to reconstruct 3D voxel. Fig. 6 shows the total processing time for each method. The general method took the longest processing time compared to lookup table and our proposed method. The look-up table method is slightly faster than our proposed method. However, the look-up table used up more memory storage compared with our proposed method.

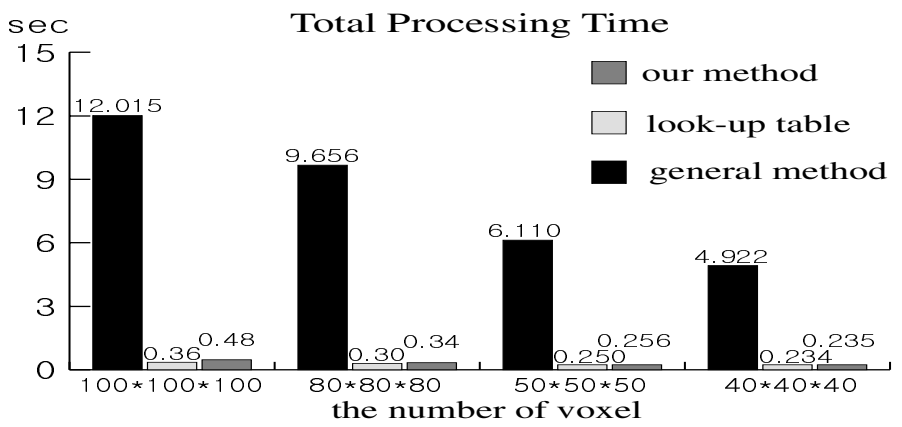

Fig. 6. Total processing time for proposed method and other existing methods

We have explained the theoretical method of meshless parameterization for existing approach and proposed approach which is integrated in $3 \mathrm{D}$ voxel reconstruction. In this experiment section, we evaluated the similarity of the proposed 
approach with the original approach. There are 3 virtually created 3D opened cubes in $5 \times 5 \times 5,7 \times 7 \times 7$ and $11 \times 11 \times 11$ size of voxel for validation purposes. The $3 \mathrm{D}$ opened cubes voxel are saved as binary format file. Fig. 7 shows the result of meshless parameterization for $5 \times 5 \times 5$ size of $3 \mathrm{D}$ opened cube, Fig. $7(\mathrm{a})$ is the result of original approach and Fig. 7(b) is the result of proposed approach.
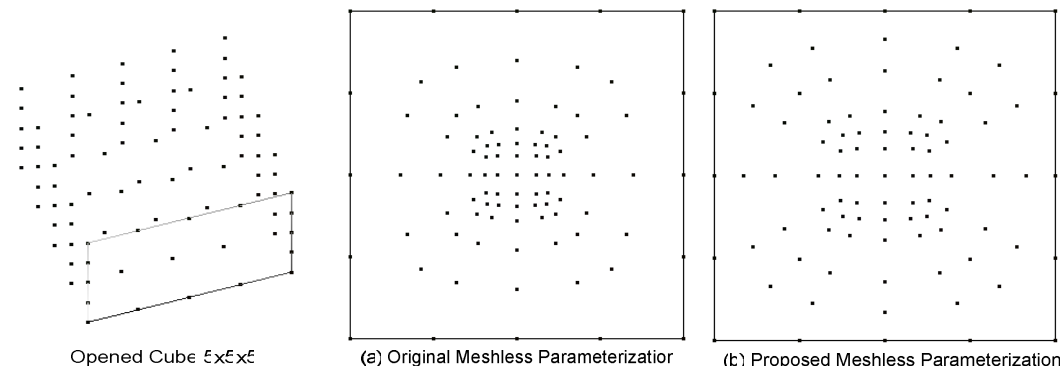

Fig. 7. Results of meshless parameterization for both approaches on $5 \times 5 \times 5$ voxel size of $3 \mathrm{D}$ opened cube

Table 2 shows the results of error difference between the proposed method and the existing method. The absolute error is used to measure the similarity of the proposed approach with the existing one. The results concluded that when the number of voxel point increase, the average or absolute error is getting lesser. And the average of minimum distance is computed between the results of $2 \mathrm{D}$ parametric. It also indicated that the average of minimum distance is reduced corresponding to the number of voxel size.

Table 2. Results of error difference between existing and proposed method

\begin{tabular}{|c|c|c|}
\hline \multirow{2}{*}{$\begin{array}{l}3 \mathrm{D} \text { opened cube } \\
(\text { volume size })\end{array}$} & \multicolumn{2}{|c|}{ Measurement of error } \\
\cline { 2 - 3 } & Average of absolute Error & Average of minimum distance \\
\hline $5 \times 5 \times 5$ & 0.00225052 & 0.141804 \\
\hline $7 \times 7 \times 7$ & 0.00108899 & 0.031601 \\
\hline $11 \times 11 \times 11$ & 0.00063468 & 0.025904 \\
\hline
\end{tabular}

In meshless parameterization method, we need to solve large linear system and compute the inverse matrix according to the number of interior points. Solving system of linear equations $A u=b$, where $A$ is a matrix of weight $n \times n, u$ is parameter points and $b$ is the sum of neighbor points of $u$, the inverse of $A^{-1}$ for matrix $A$ can be determined in time of $\Theta\left(n^{3}\right)$. As a result, the existing method of meshless parameterization is considered all number of interior points to solve the linear system. The inverse of $A^{-1}$ for matrix $A$ consumes much running time since the all number of interior points are counted. 
As our proposed approach, the meshless parameterization is executed for every three slices of 3D voxel reconstruction. It has less running time since the numbers of interior points are reduced from all points to some number of points. The inverse of matrix $A$ in our proposed approach can be determined in time of $\Theta\left((n-g)^{3}\right)$, where $g<n$. The parameter $g$ is a large number of interior points which are not included in derived slices. In this approach, we solved the running time complexity and reduced the memory space for computation process.

In addition, this approach results two tasks in one system, we adopted the advantage of 3D voxel reconstruction approach to performance the dimension reduction using meshless parameterization. This approach solved the sparse linear system and is able to maintain the existing 3D information data for any related applications.

\section{Conclusions and Future Work}

Our primary aim is to reduce the $3 \mathrm{D}$ voxel complexity without loss the information generality and satisfy the critical requirements of speed and robustness in representing $3 \mathrm{D}$ object for related applications. This paper presented a solution of the large sparse linear system of meshless parameterization, which is integrated in pre-computing 3D voxel reconstruction. In addition to this system, we proposed an efficient and fast method for reconstruct the $3 \mathrm{D}$ voxel in real time.

The proposed method using look-up table and post-computing to reconstruct 3D voxel shows the total processing time nearly closed to look-up table method. However, our proposed method is wisely reduced memory storage compared to lookup table. And for the inverse matrix of meshless parameterization operation, the running time needs $\Theta\left((n-g)^{3}\right)$, where $g<n$ and parameter $g$ is almost closed to $n$. Therefore, the running time is much lesser than existing approach. The integration of $3 \mathrm{D}$ reconstruction and dimension reduction makes this system not only efficient in term of time processing, it also wisely reduce the memory usage. Therefore, this method promises a better performance in any real time applications.

For future work, more experiments need to carry out on various kinds of 3D object to test and validate the robustness and efficiency of proposed system. The object recognition classification remains to be performed in order to judge our proposed $3 \mathrm{D}$ voxel reconstruction and meshless parameterization for dimensional reduction are not limited the shape preserving information.

Acknowledgments. This work was supported by the Soongsil University Research Fund.

\section{References}

1. Matsuyama, T., Wu, X., Takai, T., Wada, T.: Real-Time Dynamic 3D Object Shape Reconstruction and High-Fidelity Texture Mapping for 3D Video. IEEE Transactions on Circuits and Systems for Technology 14(3), 357-369 (2004)

2. Kong, G., Kanade, T., Bouguet, J., Holler, M.: A Real Time System for Robust 3D Voxel Reconstruction of Human Motion. In: Proceedings IEEE Conference on Computer Vision and Pattern Recognition, Hilton Head Island, SC, USA, vol. 2, pp. 714-720 (2000) 
3. Van der Maaten, L.J.P., Postma, E.O., Van den Herik, H.J.: Dimensionality Reduction: A Comparative Review (2007)

4. Floater, M.S.: Meshless Parameterization and B-spline Surface Approximation. In: Cipolla, R., Martin, R. (eds.) The Mathematics of Surfaces IX, pp. 1-18. Springer, Heidelberg (2000)

5. Floater, M.S., Reimers, M.: Meshless Parameterization and Surface Reconstruction. Computer Aided Geometric Design, 77-92 (2001)

6. Floater, M.S., Hormann, K.: Surface Parameterization: a Tutorial and Survey. Advances in Multiresolution for Geometric Modelling, 157-186 (2004)

7. Lee, Y., Kyoung, D., Han, E., Jung, K.: Dimension Reduction in 3D Gesture Recognition Using Meshless Parameterization. In: Chang, L.-W., Lie, W.-N., Chiang, R. (eds.) PSIVT 2006. LNCS, vol. 4319, pp. 64-73. Springer, Heidelberg (2006)

8. Voledine, T., Roose, D., Van der straeten, D: Efficient Triangulation of Point Clouds using Floater Parameterization, Report TW385 (2004)

9. Jain, R., Kasturi, R., Schunck, B.G.: Machine Vision. McGraw-Hill, Inc., New York (1995)

10. Jolliffe, I.: Principal component analysis. Technical report, Springer (October 2002)

11. Kruskal, J.B., Wish, M.: Multidimensional Scaling, Ch. 1, 3, 5, pp. 7-19, 48, 73. Sage Publications Inc., Newbury Park, CA (1978)

12. Kyoung, D., Lee, Y., Beak, W., Han, E., Yang, J., Jung, K.: Efficient 3D Voxel Reconstruction using Precomputing Method for Gesture Recognition. In: First Korea Japan Joint Workshop on Pattern Recognition (2006)

13. GML C++ Camera Calibration Toolbox, http://research.graphicon.ru/calibration/gml-c++cameracalibration-toolbox-3.html 